\title{
Hypertriglyceridemia with Acute Pancreatitis in Pediatric Diabetic Ketoacidosis: A Case Report
}

\author{
Priyank J. Yagnik ${ }^{1}$, Pooja H. Desai ${ }^{1}$, Vinai M. Modem ${ }^{2}$ \\ Science Center at Houston, Frisco, USA \\ Corresponding author: Priyank J. Yagnik, drpriyank5969@gmail.com
}

1. Pediatrics, University of Kansas School of Medicine - Wichita, Wichita, USA 2. Pediatrics, University of Texas Health

\begin{abstract}
A 16-year-old female with new-onset diabetic ketoacidosis (DKA) developed acute pancreatitis and hypertriglyceridemia within 24 hours after admission. Her insulin regimen was continued after resolution of DKA, and her pancreatitis with hypertriglyceridemia showed resolution. We are presenting a case of pediatric DKA with hypertriglyceridemia and pancreatitis treated with extended insulin.
\end{abstract}

Categories: Endocrinology/Diabetes/Metabolism, Pediatrics

Keywords: diabetic ketoacidosis complications, hyperlipidemia, milky serum, plasmapheresis, abdominal pain, dka

\section{Introduction}

Diabetic Ketoacidosis (DKA) is one of the most common diagnoses in Pediatric Intensive Care Unit (PICU). The most fatal complication of DKA is cerebral edema, but other non-fatal complications such as hypertriglyceridemia and pancreatitis are very rare and not commonly encountered in PICU [1-2]. We present the case of a new-onset DKA complicated by hypertriglyceridemia and acute pancreatitis.

\section{Case Presentation}

A previously healthy, non-obese, 16-year-old African-American female presented to a local hospital because of difficulty in breathing. Two weeks prior to this, she was diagnosed with oral thrush and prescribed nystatin by her primary care physician. She also had polyuria, polydipsia, weight loss, and decreased energy for a few days prior to presentation. She was found to have DKA at the local community hospital (serum glucose $>500 \mathrm{mg} / \mathrm{dL}$, urine glucose $>1000 \mathrm{mg} / \mathrm{dL}$, urine ketone $>80 \mathrm{mg} / \mathrm{dL}$, capillary blood $\mathrm{pH}$ of 6.8 , and serum sodium $110 \mathrm{mEq} / \mathrm{L}$ ). She presented with moderate-to-severe dehydration and received two liters ( $\sim 30$ $\mathrm{ml} / \mathrm{kg}$ ) of crystalloid fluid bolus. She also received intravenous (IV) insulin bolus and sodium bicarbonate bolus before she was transferred to our tertiary care center.

Received 12/12/2018 Review began 12/20/2018 Review ended 01/02/2019 Published 01/08/2019

๑) Copyright 2019

Yagnik et al. This is an open access article distributed under the terms of the Creative Commons Attribution License CC-BY 3.0., which permits unrestricted use, distribution, and reproduction in any medium, provided the original author and source are credited.
Upon arrival at the PICU, she continued to exhibit severe metabolic acidosis. Her examination was remarkable for Kussmaul breathing, altered mental status responding only to painful stimuli, and signs of poor perfusion (tachycardia up to 140/min, capillary refill time of 3-4 seconds, and bilateral weak peripheral pulses). She was also noted to have skin lesions, which were multiple yellowish firm papules, around both knees and scattered over the face and trunk, as seen in Figure 1. Point-of-care serum sodium level was 122 $\mathrm{mEq} / \mathrm{dL}$ but laboratory could not perform any tests on the sample since it was "too thick". It was difficult to obtain a blood sample from the peripheral IV line and once obtained, it would turn "milky". Her serum sodium showed a downward trend despite improvement in her metabolic acidosis. The possibility of hyperlipidemia was considered based on the appearance of her blood sample. Her serum triglyceride (TG) level was found to be $930 \mathrm{mg} / \mathrm{dL}$ and total cholesterol $332 \mathrm{mg} / \mathrm{dL}$. 


\section{Cureus}

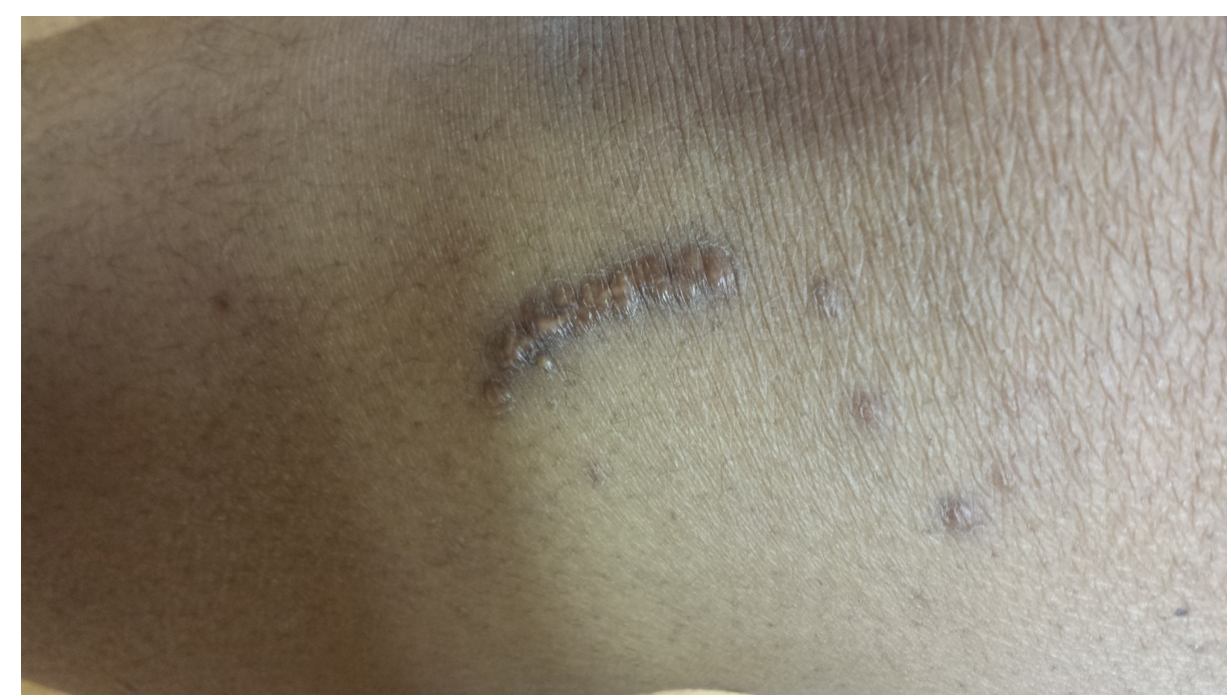

FIGURE 1: Skin lesions - multiple yellowish firm papules around the bilateral knees and scattered around the face and trunk

She was continued on IV insulin drip and twice maintenance IV fluids. Her mental status improved eight to ten hours after the admission, along with stabilization of serum glucose, but developed diffused abdominal pain with anorexia 12 hours after getting admitted to PICU. Her serum amylase was 155 unit/L and lipase 1100 unit/L. Abdominal ultrasound was consistent with acute pancreatitis and ruled out acute appendicitis, gall stone, and pancreatic pseudocyst. Between 12 to 36 hours after admission, her highest serum TG level was noted as $2515 \mathrm{mg} / \mathrm{dL}$, highest serum amylase as $612 \mathrm{unit} / \mathrm{L}$, and highest serum lipase as 5387 unit/L. Due to pancreatitis and lack of appetite, she was continued on insulin drip and maintenance IV fluid even after resolution of her metabolic acidosis. Serum glucose was measured every two hours and there was no hypoglycemic episode. Serum levels of amylase, lipase, and TG were checked every 12 hours during her PICU admission. Approximately 48 hours after admission, her appetite improved along with downward trending of serum TG (614 mg/dL) and serum lipase (574 unit/L). She was started on fenofibrate for hyperlipidemia. Dermatology was consulted for skin lesions, and the pathology report confirmed xanthoma.

\section{Discussion}

DKA is a life-threatening but preventable complication of type I diabetes mellitus. It is characterized by severe insulin deficit leading to hyperglycemia, hyperosmolar dehydration, and accumulation of ketones in the serum. Cerebral edema is encountered in about $1 \%$ cases of pediatric DKA but accounts for more than 20\% mortality in DKA [1]. Hyperlipidemia and acute pancreatitis are also among the rare complications of DKA that are not life-threatening.

Hyperglycemia is the common etiology of hyponatremia in DKA patients. Due to the osmotic flux, hyperglycemia causes shift of water from intracellular compartment to extracellular compartment, leading to dilutional hyponatremia. The corrected serum sodium in such cases is calculated by the following equation: corrected sodium $=$ measured sodium $+[1.6($ glucose -100$) / 100]$. Moreover, hyperlipidemia causes displacement of water-containing sodium, leading to pseudohyponatremia. A more accurate sodium can be measured after ultracentrifugation of the sample [3].

The insulin deficiency in type I diabetes mellitus causes hypertriglyceridemia. Insulin deficiency decreases the activity of lipoprotein lipase, which is responsible for the conversion of triglyceride to fatty acid. The triad of DKA, hyperlipidemia (specifically hypertriglyceridemia), and acute pancreatitis have been well described in adult literature. In an adult study conducted by Nair et al., hypertriglyceridemia (> $500 \mathrm{mg} / \mathrm{dl}$ ) and radiographic evidence of acute pancreatitis were found in $11 \%$ and $22 \%$ of the patients, respectively, when 100 adult patients with DKA were studied [4].

The association of DKA with hypertriglyceridemia and elevated serum amylase/lipase has also been described in the pediatric population. In a study published in 2004 involving 50 children with DKA, the authors found $40 \%$ of the patients presented with hypertriglyceridemia (> $200 \mathrm{mg} / \mathrm{L}$ ), $38 \%$ with hyperamylasemia, and $19 \%$ with hyperlipasemia. Only one patient exhibited clinically significant acute pancreatitis [5]. Pancreatic enzyme elevation is common within 12-24 hours of treatment of pediatric DKA [2].

A review by Tsuang et al. described that a TG level of $>1000 \mathrm{mg} / \mathrm{L}$ is required to ascribe the causation of acute pancreatitis. Severe hypertriglyceridemia has been treated with insulin, heparin and/or 
plasmapheresis, but comparative trials are lacking. A consensus has been established to bring a serum TG level of $<500 \mathrm{mg} / \mathrm{L}$ for symptomatic relief. Insulin activates lipoprotein lipase, and heparin stimulates the release of lipoprotein lipase from endothelium, leading to TG degradation. Plasmapheresis is a symptomatic treatment that acutely decreases the serum level of TG, thereby reducing the viscosity of blood. Oral antihyperlipidemics are recommended in cases where patients can tolerate an oral diet. Among these diets, fibrates can lower TG by $40 \%-60 \%$ [6].

The first case report of diabetic hypertriglyceridemia (serum TG $7120 \mathrm{mg} / \mathrm{dL}$ ) being successfully treated by plasmapheresis was described in 1978 [7]. Lutfi et al. described the first pediatric case of DKA with severe hypertriglyceridemia (serum TG > $16000 \mathrm{mg} / \mathrm{dL}$ ) that was successfully treated with one course of plasmapheresis. In this case report, plasmapheresis was indicated not only for hypertriglyceridemia but also for worsening abdominal pain, acute kidney injury (serum creatinine $1.2 \mathrm{mg} / \mathrm{dL}$ ), and development of pleural effusion. Plasmapheresis resulted in the significant improvement of abdominal pain along with a decrease in the serum TG level from > $5000 \mathrm{mg} / \mathrm{L}$ to $1000 \mathrm{mg} / \mathrm{L}$. The patient was started on fenofibrate upon discharge as well [8].

The patient described in the current case report was continued on IV insulin drip upon admission to PICU and then administered subcutaneous insulin after her appetite improved, approximately 48 hours after admission. IV insulin drip was continued even after resolution of her metabolic acidosis. Her serum amylase, lipase, and TG levels started trending down with insulin therapy alone. After being transferred to the floor, she was started on fenofibrate for hypertriglyceridemia. A follow-up visit two weeks after this episode showed a significant reduction of TG level to $170 \mathrm{mg} / \mathrm{dl}$. Dermatology concluded her skin lesion to be xanthoma after the biopsy. At the time of this writing, the patient was being evaluated for familial hypercholesterolemia.

\section{Conclusions}

Elevation of serum amylase and lipase are common in DKA patients. Clinically significant acute pancreatitis can be attributed to severe hypertriglyceridemia if the serum TG level is $>1000 \mathrm{mg} / \mathrm{dL}$. Treatment options include insulin, heparin, plasmapheresis, and fibrinolytic agents.

\section{Additional Information \\ Disclosures}

Human subjects: Consent was obtained by all participants in this study. Conflicts of interest: In compliance with the ICMJE uniform disclosure form, all authors declare the following: Payment/services info: All authors have declared that no financial support was received from any organization for the submitted work. Financial relationships: All authors have declared that they have no financial relationships at present or within the previous three years with any organizations that might have an interest in the submitted work. Other relationships: All authors have declared that there are no other relationships or activities that could appear to have influenced the submitted work.

\section{Acknowledgements}

We would like to thank Cari Schmidt, PhD and Minh Thuy Bui for reviewing and editing the final manuscript.

\section{References}

1. Edge J, Hawkins M, Winter D, Dunger D: The risk and outcome of cerebral oedema developing during diabetic ketoacidosis. Arch Dis Child. 2001, 85:16-22.

2. Quiros JA, Marcin JP, Kuppermann N, et al.: Elevated serum amylase and lipase in pediatric diabetic ketoacidosis. Pediatr Crit Care Med. 2008, 9:418-422. 10.1097/PCC.0b013e318172e99b

3. Oh G, Anderson S, Tancredi D, Kuppermann N, Glaser N: Hyponatremia in pediatric diabetic ketoacidosis: reevaluating the correction factor for hyperglycemia. Arch Pediatr Adolesc Med. 2009, 163:771-772. 10.1001/archpediatrics.2009.106

4. Nair S, Yadav D, Pitchumoni CS: Association of diabetic ketoacidosis and acute pancreatitis: observations in 100 consecutive episodes of DKA. Am J Gastroenterol. 2000, 95:2795-2800. 10.1111/j.15720241.2000.03188.x

5. Haddad NG, Croffie JM, Eugster EA: Pancreatic enzyme elevations in children with diabetic ketoacidosis . J Pediatr. 2004, 145:122-124. 10.1016/j.jpeds.2004.03.050

6. Tsuang W, Navaneethan U, Ruiz L, Palascak JB, Gelrud A: Hypertriglyceridemic pancreatitis: presentation and management. Am J Gastroenterol. 2009, 104:984-991. 10.1038/ajg.2009.27

7. Betteridge DJ, Bakowski M, Taylor KG, Reckless JPD, de Silva SR, Galton DJ: Treatment of severe diabetic hypertriglyceridaemia by plasma exchange. Lancet. 1978, 311:1368. 10.1016/S0140-6736(78)92450-9

8. Lutfi R, Huang J, Wong HR: Plasmapheresis to treat hypertriglyceridemia in a child with diabetic ketoacidosis and pancreatitis. Pediatrics. 2012, 129:195-198. 10.1542/peds.2011-0217 